\title{
The evolution of the jet from Herbig Ae star HD 163296 from 1999 to 2011
}

\author{
H. M. Günther ${ }^{1}$, P. C. Schneider ${ }^{2}$, and Z.-Y. Li ${ }^{3}$ \\ ${ }^{1}$ Harvard-Smithsonian Center for Astrophysics, 60 Garden Street, Cambridge, MA 02138, USA \\ e-mail: hguenther@cfa.harvard.edu \\ 2 Universität Hamburg, Hamburger Sternwarte, Gojenbergsweg 112, 21029 Hamburg, Germany \\ 3 Department of Astronomy, University of Virginia, PO Box 400325, Charlottesville, VA 22904, USA
}

Received 21 November 2012 / Accepted 12 February 2013

\section{ABSTRACT}

\begin{abstract}
Young A and B stars, the so-called Herbig Ae/Be stars (HAeBe), are surrounded by active accretion disks and drive outflows. We study the jet HH 409, which is launched from the HAeBe star HD 163296, using new and archival observations from Chandra and HST/STIS. In X-rays we can show that the central source is not significantly extended. The approaching jet, but not the counterjet, is detected in $\operatorname{Ly} \alpha$. In addition, there is red-shifted $\operatorname{Ly} \alpha$ emission extended in the same direction as the jet, which is also absent in the counterjet. We can rule out an accretion or disk-wind origin for this feature. In the optical we find the knots B and B2 in the counterjet. Knot B has been observed previously, so we can derive its proper motion of $(0.37 \pm 0.01)^{\prime \prime} \mathrm{yr}^{-1}$. Its electron density is $3000 \mathrm{~cm}^{-3}$, thus the cooling time scale is only a few months, so the knot needs to be reheated continuously. The shock speed derived from models of $\mathrm{H} \alpha$ and forbidden emission lines decreased from $50 \mathrm{~km} \mathrm{~s}^{-1}$ in 1999 to $30 \mathrm{~km} \mathrm{~s}^{-1}$ in 2011 because the shock front loses energy as it travels along the jet. Knot B2 is observed at a similar position in 2011 to where knot B was in 1999, but shows a lower ionization fraction and higher mass loss rate, proving that there are variations in the jet launching conditions.
\end{abstract}

Key words. circumstellar matter - stars: formation - stars: pre-main sequence - X-rays: stars - stars: individual: HD 163296

\section{Introduction}

Stars form when molecular clouds fragment and contract to protostars. Mass accretion onto those stellar cores proceeds by means of an accretion disk, while the surrounding envelope disperses. Eventually, the stars become optically visible; at this stage the low-mass population is called classical $\mathrm{T}$ Tauri stars (CTTS), and the A and B star progenitors are Herbig Ae/Be stars $(\mathrm{HAeBe})$. The angular momentum accreted from the disk can be removed by outflows, which come in different types. There are slow, wide-angle winds, often seen in molecular lines, such as $\mathrm{H}_{2}$, faster winds, showing up in optical forbidden emission lines (FELs), such as [O I], and highly collimated jets, which often reach velocities up to $400 \mathrm{~km} \mathrm{~s}^{-1}$ (Eislöffel \& Mundt 1998). In very young objects these jets can be traced out to a few parsecs and are still collimated well. Internal working surfaces and bow shocks are visible in emission lines as knots, the so-called Herbig-Haro $(\mathrm{HH})$ objects. The evidence that X-rays come from outflows is relatively recent, starting with $\mathrm{HH} 2$ (Pravdo et al. 2001; Schneider et al. 2012) and HH 154 (Bally et al. 2003; Favata et al. 2006; Schneider et al. 2011). In both cases the jet-driving sources are deeply embedded.

To date, we know four CTTS or HAeBe with spatially extended X-ray emission: the CTTS DG Tau (Güdel et al. 2005, 2008, 2011; Schneider \& Schmitt 2008; Günther et al. 2009), RY Tau (Skinner et al. 2011), Z CMa, a young star in an FUOr outburst phase (Stelzer et al. 2009), and the HAeBe HD 163296 (Swartz et al. 2005).

Jets from CTTS consist of layers with different velocities, where the innermost and most collimated component is the fastest one (Bacciotti et al. 2000) with velocities of a few hundred $\mathrm{km} \mathrm{s}^{-1}$. The outer layers are slower and less collimated. Often there is an asymmetry between the jet and the counterjet.
Little is known about the physical mechanism that drives the outflows. Different theoretical models of stellar winds (Kwan \& Tademaru 1988; Matt \& Pudritz 2005), X-winds (Shu et al. 1994) and disk winds (Blandford \& Payne 1982; Anderson et al. 2005) have been proposed. Ultimately, the jet launching must be powered by the gravitational energy released in the accretion process. This is supported by the observation that the outflow rate is roughly one tenth of the accretion rate (Cabrit et al. 1990; Hartigan et al. 1995; Coffey et al. 2008), but it is unclear how the energy is converted.

All models of jet launching and collimation rely on magnetic fields of some kind. However, jets are observed not only from solar-mass CTTS, but also from HAeBes. In contrast to CTTS with magnetic fields typically in the $\mathrm{kG}$ range, $\mathrm{HAeBes}$ are not expected to possess outer convection zones, so they cannot drive a solar-like dynamo, although primordial magnetic fields might be present. Often no fields or only weak ones can be observed (Hubrig et al. 2007; Wade et al. 2007): the formal limit for HD 163296 is $-25 \pm 27 \mathrm{G}$ (Hubrig et al. 2007).

In this article we present new results on the time evolution of the jet from the HAeBe HD 163296. In Sect. 2 we summarize previous work done on HD 163296 and its jet HH 409. In Sect. 3 we give details on observations and data reduction. The results from the individual observations are given in Sect. 4, followed by a discussion (Sect. 5). We end with a summary in Sect. 6 .

\section{HD 163296 and its jet $\mathrm{HH} 409$}

HD 163296 is surrounded by an accretion disk with a mass accretion rate of $\dot{M}_{\text {acc }} \approx 10^{-7} M_{\odot} \mathrm{yr}^{-1}$. This disk shows a position angle of $(140 \pm 5)^{\circ}$ and an inclinaton of $i=(60 \pm 5)^{\circ}$ 
Table 1. Log of observations.

\begin{tabular}{lccccccc}
\hline \hline Facility & Instrument & Id & Exposure & Date & PA & Central wavelength & Aperture \\
\hline Chandra & ACIS-S & 3733 & $20 \mathrm{ks}$ & $2003-08-10$ & & & \\
Chandra & ACIS-S & 12359 & $46 \mathrm{ks}$ & $2011-02-09$ & & & \\
HST & STIS/G750L & O5FO22010 & $1140 \mathrm{~s}$ & $1999-08-09$ & $43.06^{\circ}$ & $7751 \AA$ & $52 \times 0.2 \mathrm{~F} 1$ \\
HST & STIS/G140M & O57Z03010 & $2230 \mathrm{~s}$ & $1999-09-30$ & $45.15^{\circ}$ & $1218 \AA$ & $52 \times 0.2$ \\
HST & STIS/G140M & O66Q02010 & $2282 \mathrm{~s}$ & $2000-07-22$ & $46.25^{\circ}$ & $1218 \AA$ & $52 \times 0.5$ \\
HST & STIS/G140M & O66Q03010 & $7707 \mathrm{~s}$ & $2000-07-21$ & $46.50^{\circ}$ & $1218 \AA$ & $52 \times 0.2$ \\
HST & STIS/G750M & OBI801010 & $2275 \mathrm{~s}$ & $2011-07-21$ & $44.06^{\circ}$ & $6581 \AA$ & $52 \times 0.2 \mathrm{~F} 1$ \\
HST & STIS/G140M & OBI801020 & $2700 \mathrm{~s}$ & $2011-07-21$ & $44.00^{\circ}$ & $1218 \AA$ & $52 \times 0.2 \mathrm{~F} 1$ \\
\hline
\end{tabular}

(Garcia Lopez et al. 2006) ${ }^{1}$. Perpendicular to it lies a chain of nebulosities (position angle $(42.5 \pm 3.5)^{\circ}$ Grady et al. 2000), called HH 409. This jet is detected both by coronagraphic imaging (Grady et al. 2000; Wassell et al. 2006) and in HST/STIS long-slit spectra (Devine et al. 2000).

The distance to HD 163296 is $119_{-10}^{+12}$ pc (van Leeuwen 2007). The presence of a late-type companion can be ruled out with a high degree of confidence from the HST imaging, the UV and optical spectra (Swartz et al. 2005, and references therein), and more recent high-resolution optical spectroscopy is also fully consistent with HD 163296 being single (Montesinos et al. 2009).

The jet can be traced down to 0.'06 (7.1 AU) in the HST/STIS image and out to $\approx 4000 \mathrm{AU}$ in narrow band optical imaging (Wassell et al. 2006). Jet and counterjet are asymmetric. They have a different number of knots and different opening angles, but surprisingly the mass loss rate agrees better than a factor of 2 on both sides $\left(\dot{M}_{\text {jet }} \approx 10^{-8} M_{\odot} \mathrm{yr}^{-1} \approx 0.1 \times \dot{M}_{\text {acc }}\right)$. The opening angle is about $2^{\circ}$ for the jet; the initial value for the counterjet is $5^{\circ}$, but it is more collimated farther out (Wassell et al. 2006). The total space velocities calculated from proper motion and radial velocity are $360 \mathrm{~km} \mathrm{~s}^{-1}$ and $260 \mathrm{~km} \mathrm{~s}^{-1}$, respectively. The knots of the jets radiate in $\mathrm{Ly} \alpha, \mathrm{H} \alpha$, and optical FELs, e.g., [O I], [S $\left.\mathrm{S}_{\mathrm{II}}\right]$, and [ $\left.\mathrm{N}_{\mathrm{II}}\right]$. Line ratios of the last three ions are sensitive to density and temperature and thus give estimates for the electron densities in the $\mathrm{HH}$ objects of the order $10^{3} \mathrm{~cm}^{-3}$. Wassell et al. (2006) show structural differences between the knots. In knot C the $\mathrm{H} \alpha$ and $\left[\mathrm{S}_{\mathrm{II}}\right]$ emission coincide, but $\mathrm{H} \alpha$ precedes the [S II] emission in knot A. We use the naming convention of Wassell et al. (2006) throughout this work to refer to individual knots.

HH 409 seems similar to jets from CTTS in mass loss rate, electron excitation and opening angle. However, these quantities are all measured from a jet component that moves at an intermediate velocity of $200-300 \mathrm{~km} \mathrm{~s}^{-1}$. There are two indications that the fastest component of HH 409 is more energetic. Both come from X-ray observations. First, Swartz et al. (2005) detected X-ray emission (luminosity $3 \times 10^{27} \mathrm{erg} \mathrm{s}^{-1}$ ) close to knot A at 7" (=830 AU) from the star. Second, the X-ray spectrum of the star itself shows the UV-sensitive O VII triplet, whose line ratios are not compatible with emission close to the stellar surface. This suggests X-ray emission from jet collimation shocks (within a few AU of the star). Using the observed X-ray temperature, Günther \& Schmitt (2009) estimate a flow velocity of $500 \mathrm{~km} \mathrm{~s}^{-1}$ for the fastest jet component. Nevertheless, the optical FELs and the $\operatorname{Ly} \alpha / \mathrm{H} \alpha$ ratio indicate shock speeds of 80-90 $\mathrm{km} \mathrm{s}^{-1}$ (Wassell et al. 2006), which is far too slow to produce any $\mathrm{X}$-ray emission.

\footnotetext{
All position angles in this work are measured north to east.
}

\section{Observations and data reduction}

We use data from Chandra and HST/STIS observations. In the following we describe the observations and the data reduction process for each of them. The HST data is diverse. We use new optical and far-ultraviolet (FUV) data and also archival observations. Table 1 gives a summary of the data presented in this article.

\subsection{Chandra}

The X-ray observations in Table 1 were performed with Chandra/ACIS-S in the VFAINT mode. HD 163296 was placed on the back-illuminated chip S3, because this chip has the highest sensitivity for soft X-rays. This ACIS chip has a spatial resolution of $0.492^{\prime \prime}$ per pixel and an intrinsic energy resolution of 110-140 eV for energies below $2 \mathrm{keV}$. We reprocessed all Chandra data with CIAO 4.4 (Fruscione et al. 2006). Light curves and spectra for the central source were extracted using standard CIAO scripts. The background was determined from a large, source-free region on the same chip. Spectral fitting was done with the SHERPA fitting tool (Doe et al. 2007).

The ACIS point-spread function (PSF) is undersampled by the pixel size. Two techniques can be used to avoid aliasing on the pixel grid. Either the photon positions are randomized with offsets up to half a pixel or their positions are corrected with an energy-dependent subpixel event repositioning (EDSER, Li et al. 2004). The first method leads to a well-characterized, but artificially broadened PSF; the second method is characterized less well and has been implemented in CIAO only recently, but it increases the resolution of the resulting images directly. All images shown here were processed with the EDSER algorithm, but we verified that images with randomized pixel positions give compatible results.

The background light curves for both observations are flat, confirming that no flaring of the ambient spacecraft proton impact rate was present.

\subsection{HST/STIS}

The jet of HD 163296, HH 409, has been observed with HST in different instrument configurations. Here, we analyze archival and new STIS long-slit spectroscopy, where the slit is aligned with the jet axis. The observations cover two different wavelength ranges. The G140M grating is used for FUV spectroscopy, most importantly the hydrogen Ly $\alpha$ line, and the G750L and G750M gratings provide information on optical FELs with different wavelength resolutions. Table 1 gives details of the datasets used. In most cases the slit is narrow $\left(52^{\prime \prime} \times 0 . ' 2\right)$, thus the exact position angle is important, and only observation O66Q02010 


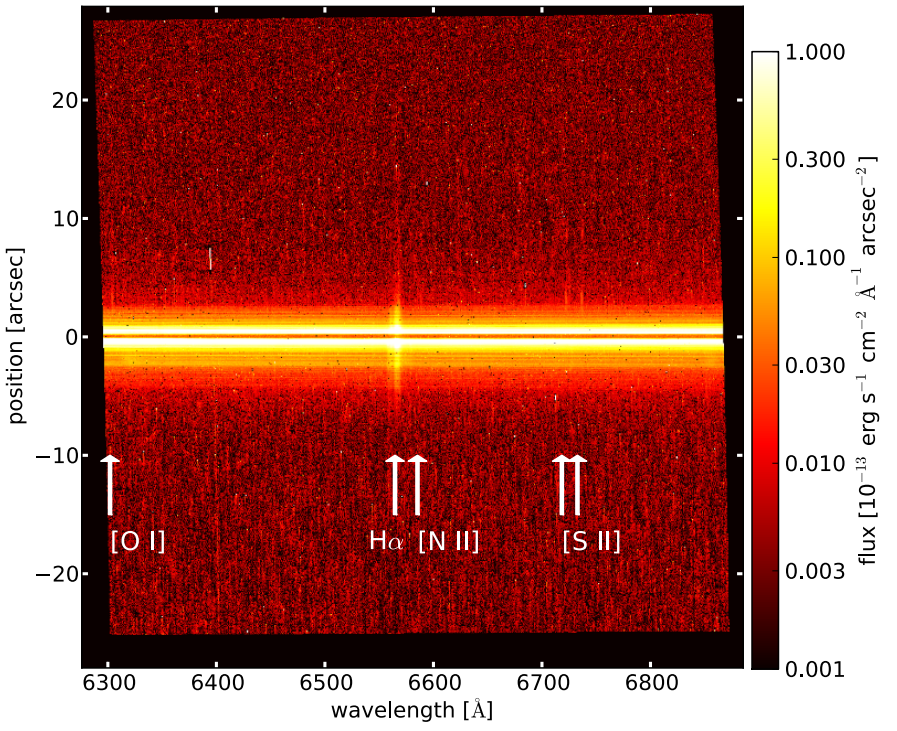

Fig. 1. G750M data from 2011. Hot pixels close to spectral lines of interest have been removed manually (see text for details). Lines which we find to be spatially extended (see Sect. 4.3) are marked.

was taken with a wider slit ( 0 ' $^{\prime} 5$ versus 0.2$)$. Grady et al. (2000) determine a jet orientation of $42.5 \pm 3.5^{\circ}$.

HD 163296 is optically bright $\left(m_{V}=6.9 \mathrm{mag}\right)$. To avoid saturation of the CCD the central star is placed behind the coronagraphic wedge (width $0.5^{\prime \prime}$, aperture $52 \mathrm{X} 0.2 \mathrm{~F} 1$ ) in all optical spectra. The same setup was chosen for our new FUV spectrum as well.

\subsubsection{New optical observations}

Pipeline processed STIS data were downloaded from MAST. A full detector image is shown in Fig. 1. Optical CCD images are corrected for overscan and bias, then dark-subtracted, flatfielded, rectified, and wavelength-calibrated to a heliocentric reference frame. The spectra span the wavelength range from approximately $6300-6865 \AA$ and the resolving power is $R \approx 5000$.

Despite the coronagraphic wedge, there is a significant stellar contribution in the optical data (Airy rings). Below we show position-velocity diagrams (PVDs) of the strongest lines in our data. To remove the stellar contribution, we define two wavelength intervals to the left and to the right of the emission line of interest (The specific wavelength regions chosen are given with the PVD.) We then estimate the stellar contribution in the region of the PVD by a linear interpolation between the left and the right wavelength regions. This procedure works because the wavelength intervals of interest are very narrow. However, low surface brightness features in the jet cannot be identified close to the central object due to the low contrast to the bright Airy rings. Furthermore, the strong, presumably stellar, $\mathrm{H} \alpha$ profile scatters into the outer jet regions and appears as extra emission in the continuum-subtracted position-velocity diagrams. A comparison with spatial profiles close to the $\mathrm{H} \alpha$ wavelength reveals that all excess flux is compatible with scattering from the central position.

\subsubsection{New FUV observations}

Due to a change in the Science Data Formatter (SDF) on HST not all events in the new G140M exposure were transferred from

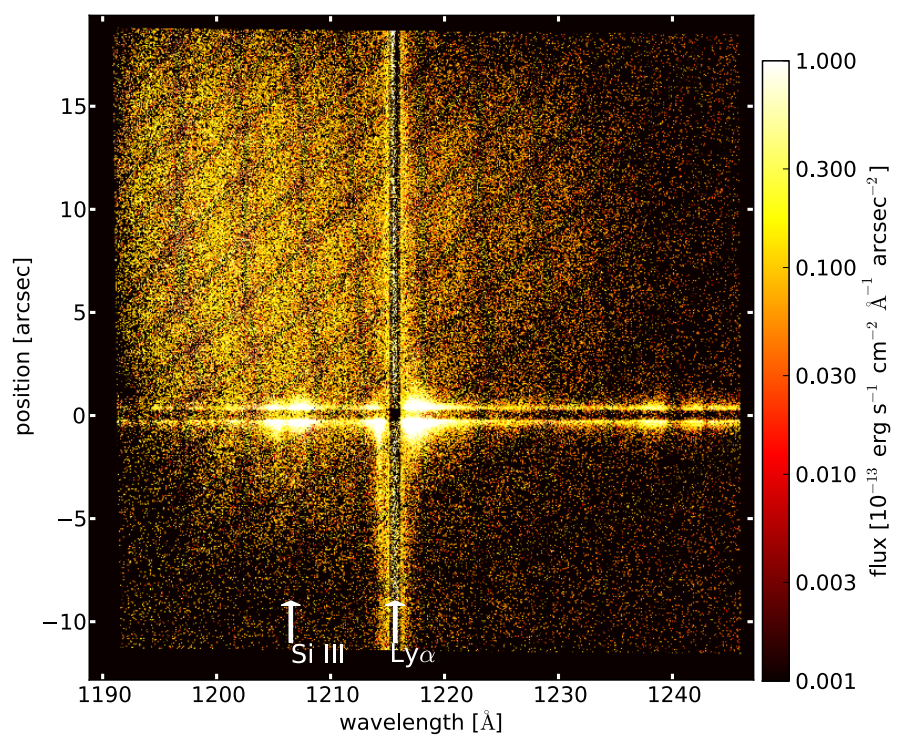

Fig. 2. G140M data from 2011. The Ly $\alpha$ line airglow feature has been removed. Lines which where found to be extended in earlier observations are marked.

the SDF to the onboard solid-state recorder (SSR) for downlink. This leads to a gap of $138.5 \mathrm{~s}$ in the exposure. Once the damaged period is removed from the telemetry stream, the resulting raw data is processed with the standard calstis pipeline in the same way as the raw data from all other observations. A full detector image is shown in Fig. 2.

CalSTIS divides the image by a flatfield, then it is rectified and wavelength-calibrated. The data covers approximately the range 1190-1245 A. We use a binning of four high-resolution pixels, which provides a wavelength resolution of $0.2 \AA$ for a point source. For comparison the effective resolution of emission that fills the slit uniformly, would be $\approx 80 \mathrm{~km} \mathrm{~s}^{-1}$.

While the overall dark current is low, the top lefthand corner of the FUV-MAMA detector shows an enhanced glow that is intrinsic to the microchannel plate array. The strength of the glow is time variable, but it can be removed by scaling archival dark exposures. However, these have a limited signal-to-noise ratio and thus the subtraction would increase the noise in the residual image. Since the glow changes on much larger spatial scales than the features of the jet, we fit the local background around the knots of the jet instead of subtracting it globally.

The FUV images are dominated by strong Ly $\alpha$ airglow emission. For each position we determine the centroid of the airglow line. We build an average line profile from regions close to the detector edges and far from the known jet emission. At each position we subtract this profile from the image. The accuracy of this procedure is limited by the statistical noise in the airglow line. We estimate that only features with a surface luminosity of $>10 \%$ of the observed airglow line can be detected. All residuals are smaller than this value. The data is taken in TIME-TAG mode, and as a cross-check we limited the analysis to times where the count rate in the airglow is less than a third of the maximum rate. We conclude that no astrophysical signal is found at the position of the airglow line.

In the FUV the Airy rings are much less severe. The generally lower count rates mean that the interpolation is not defined as well and the procedure described above would introduce significant noise to the PVDs. Therefore, no subtraction of the contribution from the unresolved source was attempted for the FUV exposures. 


\subsubsection{Archival data}

Pipeline processed STIS data were downloaded from MAST and treated in the same way as the new FUV or optical data. While all G140M observations are useful for analyzing material close to the central source, we use only the observations from 1999 and 2011 when we look at the knots of the jet, which are located a few arcsec from the star because Wassell et al. (2006) show that the position angle of $46.5^{\circ}$ in the deeper observation O66Q03010 is too large and that the slit misses the apex of the jet shocks. Observation O66Q02010 is the only dataset taken with a wider slit. While knots are visible, their flux is uncertain because they partially overlap with the strong $\operatorname{Ly} \alpha$ airglow.

\subsubsection{Data reduction}

We processed the data further with custom python routines. Hot or negative pixels were marked by hand and replaced with the average value of the surrounding pixels. The jet emission is weak both in the FUV and in the optical. Consequently, the signal-tonoise ratio $(\mathrm{S} / \mathrm{R})$ for a single pixel is typically two to four in the jet. However, all features analyzed stretch over a larger detector area. We present the data binned in the spatial direction over four pixels for the FUV and over six pixels for [O I] $6300 \AA$ and the [S II] lines at $6716 \AA$ and $6731 \AA$. When we fit fluxes and positions for knots in the jet, we achieve even better $\mathrm{S} / \mathrm{N}$ because they stretch over a range of velocities as well. Uncertainties given in the tables are $1 \sigma$ confidence intervals throughout the article.

\section{Results}

\subsection{X-ray emission}

The first Chandra observation of HD 163296 has already been presented by Swartz et al. (2005), but we analyze it here again for comparison. HD 163296 has also been observed with XMM-Newton by Günther \& Schmitt (2009).

\subsubsection{The central star}

The light curves and hardness ratios for the archival observations are moderately variable, and the new observation is fully compatible with those findings. The XMM-Newton spectra have a higher $\mathrm{S} / \mathrm{N}$ than the Chandra data because the effective area of XMM-Newton is larger, the exposure time is longer, and $X M M-N e w t o n /$ RGS delivers a high-resolution grating spectrum. We used the same spectral model for HD 163296 as in Günther \& Schmitt (2009, one phabs absorber and three vapec, optically thin, collisionally excited, thermal emission components with nonsolar abundances) and kept the abundances fixed at the values found in the XMM-Newton observation. Elements like $\mathrm{Mg}$, $\mathrm{Fe}, \mathrm{Si}$, and $\mathrm{C}$ with a low first-ionization potential (FIP) are enhanced compared to $\mathrm{O}$ and $\mathrm{Ne}$, which have a higher FIP. This is a common effect in inactive stars. Also, we kept the absorbing column density fixed at $N_{\mathrm{H}}=7 \times 10^{20} \mathrm{~cm}^{-2}$, which is in full agreement with the optical reddening (Günther \& Schmitt 2009).

Table 2 lists the temperature and volume emission measures for the three temperature components. Both Chandra observations are refit here, and the reduced $\chi^{2}$ values of both fits are below 1 . Statistically acceptable fits are possible with only two temperature components, but we kept the model of Günther \& Schmitt (2009) to facilitate a comparison. The fit values for the $X M M-N e w t o n$ observation were taken directly from that work
Table 2. Best-fit model parameters ( $1 \sigma$ confidence interval).

\begin{tabular}{|c|c|c|c|c|}
\hline \multicolumn{2}{|c|}{ Component } & Soft & Medium & Hard \\
\hline \multicolumn{5}{|c|}{ Parameters fixed for all components ${ }^{a}$} \\
\hline$N_{\mathrm{H}}$ & {$\left[10^{20} \mathrm{~cm}^{-2}\right]$} & 7 & 7 & 7 \\
\hline $\mathrm{C}$ & $b$ & 3.7 & 3.7 & 3.7 \\
\hline $\mathrm{O}$ & $b$ & 0.7 & 0.7 & 0.7 \\
\hline $\mathrm{Ne}$ & $b$ & 1.2 & 1.2 & 1.2 \\
\hline $\mathrm{Mg}$ & $b$ & 2.3 & 2.3 & 2.3 \\
\hline $\mathrm{Si}$ & $b$ & 2.8 & 2.8 & 2.8 \\
\hline $\mathrm{Fe}$ & $b$ & 1.6 & 1.6 & 1.6 \\
\hline \multicolumn{5}{|c|}{ Chandra 2003 (red. $\left.\chi^{2}=0.91\right)$} \\
\hline$k T$ & {$[\mathrm{keV}]$} & $0.19_{-0.02}^{+0.23}$ & $0.60_{-0.04}^{+0.05}$ & $2.0_{-0.5}^{+1.0}$ \\
\hline VEM & {$\left[10^{52} \mathrm{~cm}^{-3}\right]$} & $\begin{array}{l}1.3_{-0.5}^{-0.02} \\
\end{array}$ & $\begin{array}{l}0.9_{-0.2}^{+0.04} \\
\end{array}$ & $0.4_{-0.1}^{-0.5}$ \\
\hline \multicolumn{5}{|c|}{ XMM-Newton $2007^{c}$} \\
\hline$k T$ & {$[\mathrm{keV}]$} & $0.21_{-0.01}^{+0.02}$ & $0.51_{-0.02}^{+0.1}$ & $2.7_{-0.5}^{+1.0}$ \\
\hline VEM & {$\left[10^{52} \mathrm{~cm}^{-3}\right]$} & $2.2_{-0.3}^{+0.51}$ & $1.1_{-0.4}^{+0.02}$ & $0.5_{-0.2}^{-0.5}$ \\
\hline \multicolumn{5}{|c|}{ Chandra 2011 (red. $\left.\chi^{2}=0.85\right)$} \\
\hline$k T$ & [keV] & $0.16_{-0.01}^{+0.01}$ & $0.60_{-0.02}^{+0.2}$ & $2.4_{-0.3}^{+0.5}$ \\
\hline VEM & {$\left[10^{52} \mathrm{~cm}^{-3}\right]$} & $1.3_{-0.2}^{-0.01}$ & $\begin{array}{l}1.0_{-0.6}^{+0.02} \\
0.6\end{array}$ & $0.4_{-0.1}^{-0.3}$ \\
\hline
\end{tabular}

Notes. ${ }^{(a)}$ From the XMM-Newton fit. See Günther \& Schmitt (2009) for details; ${ }^{(b)}$ abundances relative to the solar values of Grevesse \& Sauval (1998); ${ }^{(c)}$ all values taken from Günther \& Schmitt (2009).

(after correction for the revised distance we use in this article). The temperatures of all three components are very similar and consistent within the errors over all three observations. The same is true for the volume emission measure (VEM) with the exception of the soft component, which seems marginally stronger in the XMM-Newton observation.

The light curves show little variability during the observations, and the comparison of the spectra and their emission measures over nearly one decade shows that the X-ray emission of the HAeBe HD 163296 is stable. The observed (absorbed) energy flux in the 2011 observations is $(2.4 \pm 0.2) \times$ $10^{-13} \mathrm{erg} \mathrm{s}^{-1} \mathrm{~cm}^{-2}(0.3-5.0 \mathrm{keV})$. From this we derive an intrinsic $\log L_{\mathrm{X}}=29.8$ (in ergs s${ }^{-1}$ ), which is almost identical to the values of previous observations (Günther \& Schmitt 2009).

\subsubsection{The base of the jet}

Based on a line ratio in the $\mathrm{O}$ VII He-like triplet that is sensitive to the ambient UV field Günther \& Schmitt (2009) conclude that the soft X-ray emission must be emitted at least $0.8 R_{*}$ above the stellar surface.

In the CTTS DG Tau, Schneider \& Schmitt (2008) directly measure an offset of about $40 \mathrm{AU}$ between the centroids of the soft and the hard components of the central source. The hard component is presumably stellar emission, while the soft component might originate in a jet shock close to the star. This detection is possible in DG Tau, because the high column density absorbs all soft X-rays from the star itself. We search for an offset using the same technique in HD 163296 but find no hint of extension. A larger physical separation would be required in HD 163296 than in DG Tau, because the absorbing column density is very low, so any stellar plasma would also contribute to the soft band flux. 

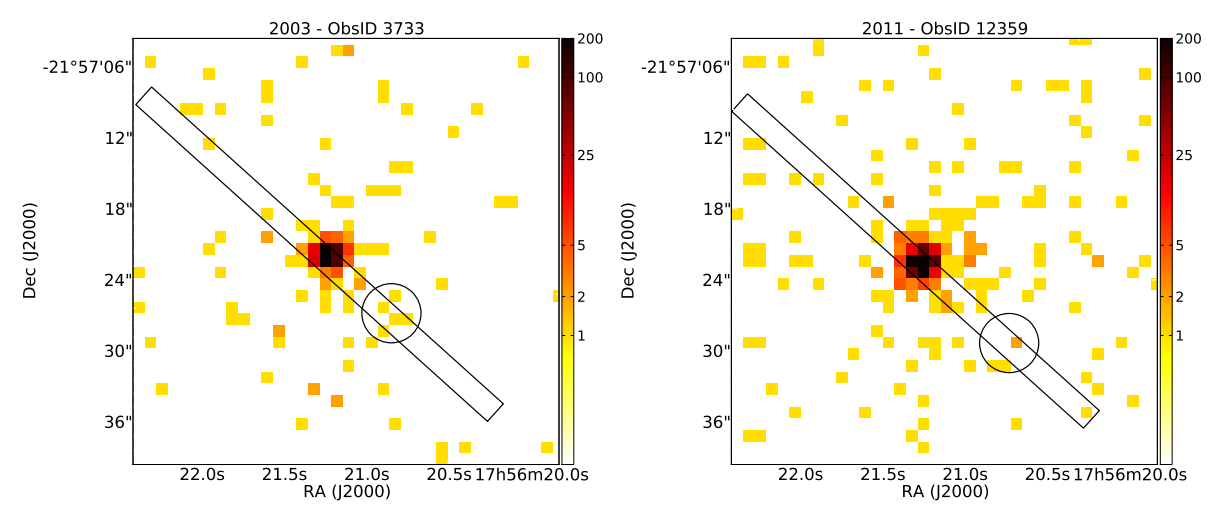

Fig. 3. Chandra/ACIS image of HD 163296 and its jet $\mathrm{HH} 409$ in the energy range $0.2-5.0 \mathrm{keV}$ binned up by a factor of two (Each block is 2 pixel $\approx 1^{\prime \prime}$ on the side). The black box shows the position of the optical jet. The half-width of the box is comparable to the PSF $\left(1^{\prime \prime}\right)$. The long axis of the box passes through the centroid of the X-ray emission and has the position angle $42^{\circ}$. Circles indicate locations of possible emission knots. The radii are $2.5^{\prime \prime}$. (This figure is available in color in electronic form.)
Additionally, we simulate the Chandra point-spread function with SAOTrace Version 2.0.1 (Carter et al. 2003). This tool performs Monte-Carlo ray-tracing through the Chandra optics. In the current version it includes the telescope dither pattern. From the ray-trace we calculate the detector response with MARX (Wise et al. 1997), version 5.0. The combination of SAOTrace and MARX can properly simulate the effect of the energydependend subpixel event repositioning. We find an extension beyond the simulated PSF in the range $0.5-0.8^{\prime \prime}$. However, a deconvolution of the observed image with the simulated PSF using the CIAO tool arestore shows that the extension in both observations is perpendicular to the jet direction. It matches the PSF asymmetry recently detected in Chandra/HRC observations (Juda \& Karovska 2010) in both the distance to the center of the PSF and in the relative position in detector coordinates. Thus, the central source itself is apparently not extended. In comparison to DG Tau, where the jet is detected within $30 \mathrm{AU}$ of the central star using image deconvolution (Güdel et al., in prep.), we conclude that almost all soft X-ray emission from HD 163296 is contained within the inner $30 \mathrm{AU}$.

\subsubsection{The X-ray knots of the jet}

Figure 3 shows the region around HD 163296 in X-rays. The position angle of the jet is well known from optical images. Thus, we can select the jet region a-priori and do not need to rely on a source detection in the Chandra images. We select events with energies in the range $0.2-2.0 \mathrm{keV}$. Figure 4 shows a projection of all counts within $1^{\prime \prime}$ of the jet axis.

In the data from 2003 the projection of the jet is above the comparison lines in the range -3 to -4 " (negative numbers are for the approaching southwest jet). To estimate the significance, we determine an average number of 1.75 counts between $-4.5^{\prime \prime}$ and $-2.5^{\prime \prime}$ from the wings of the PSF, while five counts are observed in this range along the jet direction. In a Poisson distribution the chance to observe five counts when the expectation value is 1.75 is $3 \%$. Thus, this feature is $97 \%$ significant. This does not contradict the results of Sect. 4.1.2, because this feature is two orders of magnitude less luminous than the central star and thus does not show up in an image deconvolution. This emission corresponds to the extension of the source seen by Swartz et al. (2005). A similar feature is seen along the jet in 2011 but also in directions other than the jet, thus it is not clear that this feature is present in 2011 .

In the projection the strongest features beyond the stellar PSF are two peaks at $-7.2^{\prime \prime}$ and $-10.6^{\prime \prime}$ in 2003 and 2011, respectively. Swartz et al. (2005) derive a probability of only 0.04 that the knot in 2003 (left panel of Fig. 3) is a chance fluctuation by calculating the number of circles of this size at any position

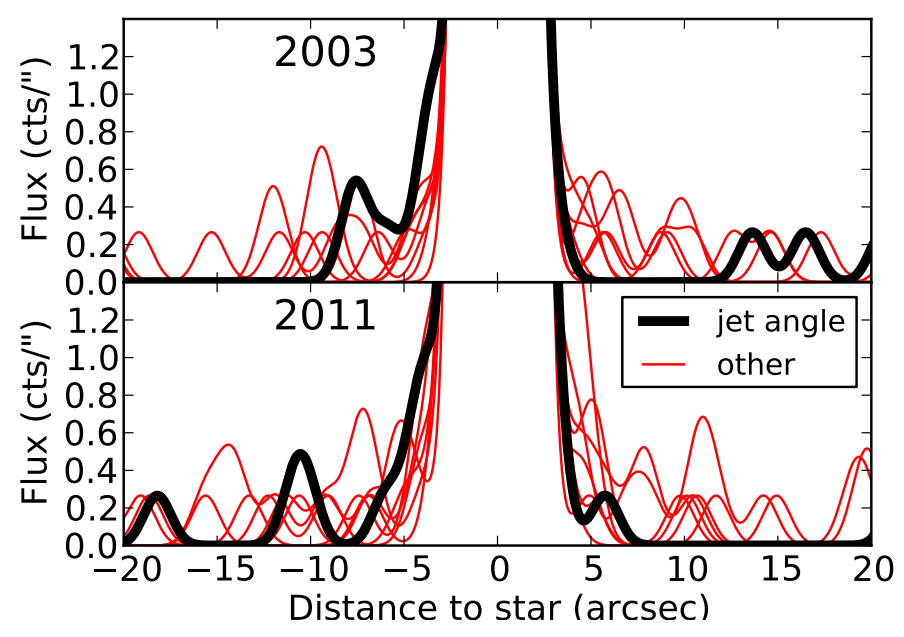

Fig. 4. Projection of Chandra/ACIS counts into one dimension. Black line: projection onto the jet axes in a rectangle with half-width $1^{\prime \prime}$. Red/gray lines: projection onto a set of lines through HD 163296, but with a set of angles rotated by $20^{\circ}, 40^{\circ}, 60^{\circ}, 80^{\circ}, 100^{\circ}, 120^{\circ}, 140^{\circ}$ and $160^{\circ}$ with respect to the jet. All fluxes are smoothed. Distances are positive for the NE jet and negative for the SW jet. See the electronic edition of the Journal for a color version of this figure. (This figure is available in color in electronic form.)

on the detector that contain five or more photons. We confirm this number using Poisson statistics of the measured background in a near-by source free region $\left(0.008\right.$ and $0.012 \mathrm{cts}^{\text {pixel }}{ }^{-1}$ in 2003 and 2011, respectively, in the energy range $0.2-2.0 \mathrm{keV}$ ). Thus, we expect 0.65 and 0.97 background events in a circle with radius $2.5^{\prime \prime}$. According to Poisson statistics the chance of finding five or more photons in any one circle in 2003 is therefore $<0.1 \%$, and the chance is $7 \%$ to find three or more photons in 2011. To estimate the significance of the detection, we need to consider the number of circles searched. We analyzed a region up to $25^{\prime \prime}$ from the central star in both jet directions, which can be covered by ten circles with radius $2.5^{\prime \prime}$. (We would recognize features that fall between two of those circles, so more than ten positions are searched. However, only the number of independent positions is important). In 2003, the probability of finding no circle with five counts or more is $99 \%$, which we can take as the significance of detecting a X-ray feature in the jet; the significance of an independent detection of the X-ray feature in the jet in 2011 is only 44\%. A potential knot with three photons in 2011 is not significant by itself. However, if we assume the X-ray feature in 2003 is real and then propagate it with the proper motion of the optical knot A, which shows $0.49 \pm 0.02 \mathrm{yr}^{-1}$ (Wassell et al. 2006), then this defines the search area exactly (Fig. 3, right panel) and the chance of finding three or more photons in a single 
Table 3. Position of knots from Gaussian fit.

\begin{tabular}{lccc}
\hline \hline Knot & Tracer & Epoch & Position \\
\hline X-ray knot & X-ray & 2003.69 & $-7.2 \pm 1.0^{\prime \prime a}$ \\
X-ray knot & X-ray & 2011.11 & $-10.6 \pm 0.3^{\prime \prime a}$ \\
& Ly $\alpha$ & 2011.64 & $\approx-2^{\prime \prime}$ \\
& Ly $\alpha$ & 2011.64 & $-4.0 \pm 0.2^{\prime \prime}$ \\
knot B & {$\left[\mathrm{S}_{\text {II }} 6731 \AA\right.$} & 1999.69 & $4.3 \pm 0.1^{\prime \prime}$ \\
knot B & {$\left[\mathrm{S}_{\text {II }} 6731 \AA\right.$} & 2011.64 & $8.7 \pm 0.1^{\prime \prime}$ \\
knot B2 & {$\left[\mathrm{S}_{\text {II }} 6731 \AA\right.$} & 2011.64 & $3.3 \pm 0.1^{\prime \prime}$ \\
\hline
\end{tabular}

Notes. ${ }^{(a)}$ The uncertainty is calculated from the variance of the photon position for all photons attributed to the knot.

circle of this size is $<7 \%$. Thus, we can say that if the knot in 2003 is real and has the same proper motion as the optical knots, we detect an X-ray knot in 2011 with $93 \%$ significance.

We note that the half-width of the detection region used by Swartz et al. (2005) is 2.5", much greater than expected from the width of the optical knots and the Chandra PSF. Figure 4 shows the projection of the counts on the jet axis with an extraction region matched to the PSF. Several features in the comparison sample (projected on lines that do not coincide with the jet axis) have similar fluxes to the tentative knots along the jet. However, in HH 2 Schneider et al. (2012) find that the X-ray emitting region is larger than the optical knots. If the same is true here, then it is not surprising that the knots are only detected if we use a large extraction region.

The effective area of the Chandra/ACIS detector degraded between 2003 and 2011 (about $5 \%$ at $1 \mathrm{keV}, 20 \%$ at $0.8 \mathrm{keV}$ and $40 \%$ at $0.5 \mathrm{keV}$ ), but the observation taken in 2011 is more than twice as long. Taking the three photons at $-10.6^{\prime \prime}$ as an upper limit to the flux of the tentative X-ray knot, the apparent luminosity decreased by a factor three compared to 2003. Although the count number is low, the hypothesis that the photon flux is the same in both observations can be rejected on the $95 \%$ level.

In summary, we can say that in 2003 an X-ray knot is detected with $95 \%$ significance, confirming Swartz et al. (2005). Under the assumption that this knot moves with a proper motion similar to the optical knots (as observed in HH 2 Schneider et al. 2012), this feature is detected again in 2011, but with lower luminosity.

\subsubsection{Summary of $X$-ray results}

HD 163296 itself is very stable over the last decade. Lightcurves and hardness ratios in all observations are nearly flat and the temperatures and emission measures of all spectral fits agree. From the O VII line ratio Günther \& Schmitt (2009) conclude that the soft X-ray emission must originate above the stellar surface, but we do not see an offset between the soft and the hard centroids, setting an upper limit on the separation of 30 AU. However, very weak emission features (knots of the jet) are seen on the edge of the PSF at -4 " along the jet. Furthermore, there is a weak feature at $-7.2^{\prime \prime}$ in 2003 and a tentative feature at $-10.6^{\prime \prime}$ in 2011 (Table 3). Neither of these features has an optical or UV counterpart (see below).

\subsection{UV emission}

HH 409 was observed at UV wavelengths with STIS previously in 1999 and in 2000. The most prominent line in the FUV is Ly $\alpha$. Its central part is contaminated by ISM absorption and airglow, which fills the aperture. Thus, only data more than $150 \mathrm{~km} \mathrm{~s}^{-1}$ from the line center can be used. Devine et al. (2000) found blue-shifted $\operatorname{Ly} \alpha$ emission in the jet with a velocity around $380 \mathrm{~km} \mathrm{~s}^{-1}$ at $0.06^{\prime \prime}$ (7.1 AU), which decreased to $335 \mathrm{~km} \mathrm{~s}^{-1}$ at 6" (714 AU). Additionally, they could identify three knots (Knot $\mathrm{A}$ in the jet at $-7.7^{\prime \prime}$ and knots $\mathrm{B}$ and $\mathrm{C}$ in the counterjet at $4.5^{\prime \prime}$ and 8.6", respectively).

Figure 5 (left) shows the position-velocity diagram (PVD) of Ly $\alpha$ for the new observation. Despite the slightly longer exposure time, the data quality of the new observation does not reach the level of the observation in 1999.

In the jet itself there are two knot features at about $-2^{\prime \prime}$ and $-4{ }^{\prime \prime}$. At the given $\mathrm{S} / \mathrm{N}$ they are described well by a Gaussian. Fitted values for the position are given in Table 3, line fluxes in Table 4. Figure 6 shows spectra extracted at the position of the two knots. The features are in the same range of velocities and speeds as was observed by Devine et al. (2000), and it indicates that the flow speed of the jet has not changed considerably in a decade.

The Si III line at $1206.5 \AA$ is too weak to be fitted independently. Thus, we fix the line shift and width at the values found for $\operatorname{Ly} \alpha$. For the outer knot we can only give an upper limit, and for the inner one we formally find a detection (Table 4). However, we caution that the flux is weak, and the position of the inner knot coincides with a region of enhanced detector background in Si III. Thus, the flux might be overestimated.

In principle, the models of Hartigan et al. (1987) constrain the shock velocity, given the Ly $\alpha / \mathrm{Si}$ III flux ratio. These models are based on simulations of radiative shocks. The preshock material is assumed to be in ionization equilibrium with the postshock material. This describes a scenario where the Lyman radiation from the shock front is the main ionization source for the preshock gas. At a distance of a few hundred AU from the central star, this is a reasonable assumption.

In all these models the flux ratio $\operatorname{Ly} \alpha / \mathrm{Si}$ III $>10$. Thus, they are not constrained by the upper limit in the outer knot, and they cannot explain the tentative Si III detection in the inner knot. It is possible that scatter from the central star contributes to the inner emission similarly to the spatially extended Ly $\alpha$ emission, which is seen to be red-shifted on the side of the approaching jet and which possibly also contributes to the blue-shifted emission.

The observed surface brightness of the Ly $\alpha$ emission is reduced compared to 1999. This can be seen in Table 4, which shows the fluxes integrated over the visible knot surface. Either the jet is dimmer in 2011 or it has broadened considerably, so that only part of the total luminosity is contained in the aperture. The latter explanation seems unlikely, because the jet was centrally bright in previous observations. Also, in a quasi-static scenario where gas travels along the jet but the overall shape of the jet stays constant with time, this is not expected.

The line fluxes in Table 3 are not dereddened. Devine et al. (2000) found $E(B-V)=0.015$, which leads to a flux correction of $15 \%$ (Wassell et al. 2006).

Given the proper motion of the old knots A and C (Wassell et al. 2006), they should be located at $-13.6^{\prime \prime}$ and $12.7^{\prime \prime}$ during the new observation. No significant emission is observed at these locations or at the tentative X-ray knot at $-10.6^{\prime \prime}$.

After subtracting the spectrum of the unresolved source, Devine et al. (2000) find red-shifted Ly $\alpha$ emission to the southwest of the central source in the direction of the blue-shifted jet. Figure 7 shows the spatial profile of the emission on the red-shifted side of the Ly $\alpha$ line in comparison with the continuum emission, which originates in the star. The continuum and lines are normalized at $0.04^{\prime \prime}$, where the continuum reaches the maximum of its spatial profile in our spatial binning. The redshifted Ly $\alpha$ emission shows extended emission on the side of 


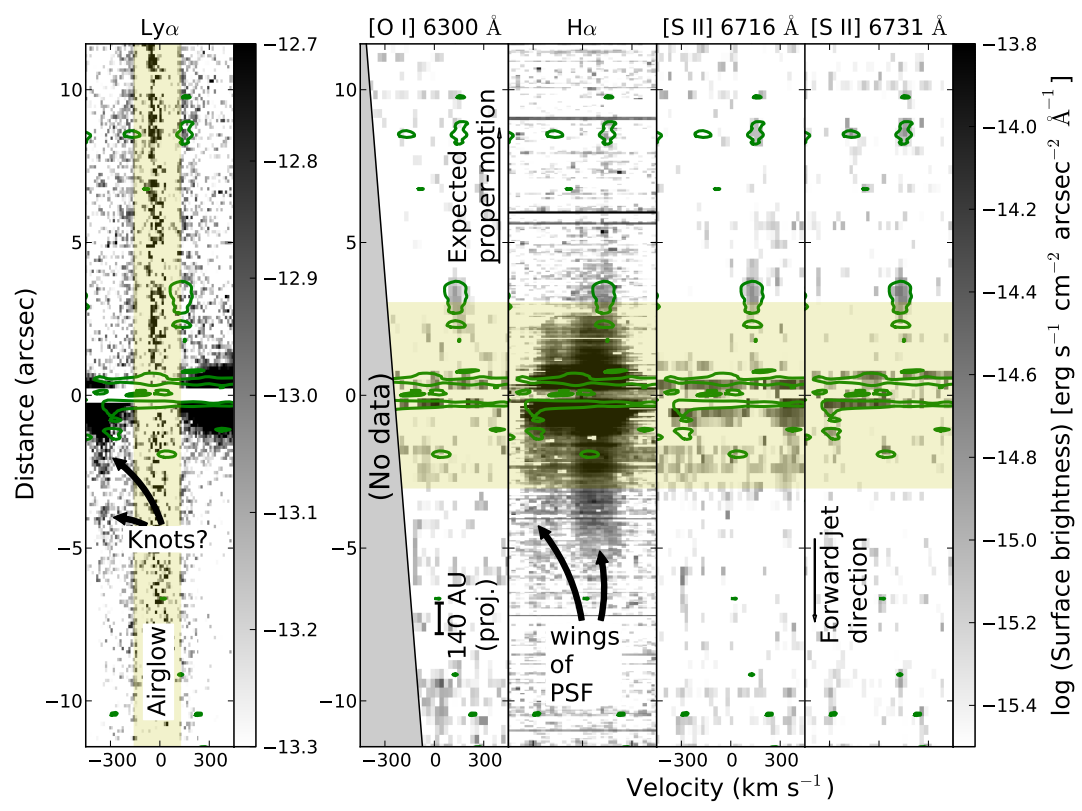

Fig. 5. PVD for $\mathrm{Ly} \alpha, \mathrm{H} \alpha$ and three optical FELs. For comparison, the contour lines for $\left[\mathrm{S}_{\mathrm{II}}\right] 6731 \AA$ are overlaid in all panels. Left: the Ly $\alpha$ line is contaminated by airglow close to the rest velocity. Right: the optical lines show scattered light close to the central star despite the occulting bar. These areas are shaded. [O I] $6300 \AA$ is located close to the edge of the chip, so no data exists in the bottom left of the PVD. Contour lines are drawn for $\left[\mathrm{S} \mathrm{II}_{\mathrm{II}} 6731 \AA\right.$ at the level $7 \times 10^{-16} \mathrm{erg} \mathrm{s}^{-1} \AA^{-1} \mathrm{~cm}^{-2} \operatorname{arcsec}^{-2}$. The optical PVDs are background-subtracted (see text). The following wavelength intervals are used to fit the background: [O I] $6300 \AA$ А: 6352-6357 , and 6376-6382 $⿱$;

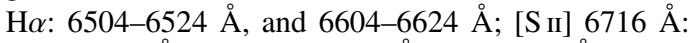
6705-6708 A, and 6721-6724 А; [S II] $6731 \AA$ : 6721$6723 \AA$, and $6735-6738 \AA$. (This figure is available in color in electronic form.)

Table 4. Fluxes in knots ( $1 \sigma$ uncertainties).

\begin{tabular}{|c|c|c|c|c|c|}
\hline Line & $\begin{array}{l}\lambda \\
\AA\end{array}$ & $\begin{array}{r}\text { Flux (1999) } \\
10^{-16} \mathrm{erg}\end{array}$ & $\begin{array}{l}\text { Flux (2011) } \\
\mathrm{s}^{-1} \mathrm{~cm}^{-2}\end{array}$ & $\begin{array}{c}v \\
\mathrm{~km} \mathrm{~s}^{-1}\end{array}$ & $\begin{array}{c}\sigma \\
\mathrm{km} \mathrm{s}^{-1}\end{array}$ \\
\hline \multicolumn{6}{|c|}{ jet: -3 to $-1^{\prime \prime}$} \\
\hline $\operatorname{Ly} \alpha$ & 1216 & $\ldots$ & $49_{-5}^{+2}$ & $353_{-3}^{+7}$ & $40_{-4}^{+2}$ \\
\hline $\mathrm{Si}$ III & 1207 & $\ldots$ & $16^{a}$ & $=353^{a}$ & $=40^{a}$ \\
\hline \multicolumn{6}{|c|}{ jet: -5.1 to $-3.3^{\prime \prime}$} \\
\hline $\operatorname{Ly} \alpha$ & 1216 & $\ldots$ & $32 \pm 2$ & $331_{-9}^{+6}$ & $77_{-2}^{+5}$ \\
\hline Si III & 1207 & $\ldots$ & $<4^{a}$ & $=331^{a}$ & $=77^{a}$ \\
\hline \multicolumn{6}{|c|}{ counterjet: B2 } \\
\hline$\left[\mathrm{O}_{\mathrm{I}}\right]$ & 6300 & $\ldots$ & $6.3 \pm 0.6$ & $130 \pm 10$ & $41_{-2}^{+5}$ \\
\hline $\mathrm{H} \alpha$ & 6563 & $\ldots$ & $8.4_{-0.1}^{+5.7}$ & $144_{-13}^{+3}$ & $50 \pm 4$ \\
\hline$\left[\mathrm{N}_{\text {III }}\right]$ & 6586 & $\ldots$ & $2.9_{-0.5}^{+0.1}$ & $127_{-10}^{+13}$ & $33_{-6}^{+13}$ \\
\hline$[\mathrm{S}$ II] & 6716 & $\ldots$ & $6.4_{-0.5}^{+0.9}$ & $132 \pm 4$ & $31 \pm 3$ \\
\hline$[\mathrm{S}$ II $]$ & 6731 & $\ldots$ & $9.1 \pm 0.6$ & $130 \pm 3$ & $35 \pm 3$ \\
\hline \multicolumn{6}{|c|}{ counterjet: B } \\
\hline$\left[\mathrm{O}_{\mathrm{I}}\right]$ & 6300 & $2.3_{-1.0}^{+1.1}$ & $2.0_{-05}^{+0.7}$ & $139_{-20}^{+3}$ & $36_{-3}^{+16}$ \\
\hline $\mathrm{H} \alpha$ & 6563 & $21 \pm 2.0^{b}$ & $2.0_{-0.3}^{+0.5}$ & $132 \pm 4$ & $20.02_{-0.01}^{+6}$ \\
\hline$\left[\mathrm{N}_{\text {III }}\right]$ & 6586 & $4.8_{-1.1}^{+1.5}$ & $1.4_{-0.3}^{+0.6}$ & $119 \pm 7$ & $25.7_{-0.3}^{+2.01}$ \\
\hline$[\mathrm{S}$ II $]$ & 6716 & $0^{+1.3}$ & $2.2_{-0.3}^{+0.3}$ & $156 \pm 7$ & $24 \pm 7$ \\
\hline$\left[\mathrm{S}_{\mathrm{II}}\right]$ & 6731 & $20_{-23}^{+1.5}$ & $3.4 \pm 0.4$ & $153_{-5}^{+3}$ & $28_{-3}^{+5}$ \\
\hline
\end{tabular}

Notes. ${ }^{(a)}$ The Si III lines are too weak to fit without constraints, thus we fix $v$ and $\sigma$ at the values found for Ly $\alpha$ at the same position; ${ }^{(b)}$ in the G750L grating used in 1999 , the $\mathrm{H} \alpha$ line is blended with $\left[\mathrm{N}_{\mathrm{II}}\right] 6548 \AA$. This contribution is subtracted assuming the quantum mechanical ratio $\left[\mathrm{N}_{\text {II }}\right] 6584 \AA /\left[\mathrm{N}_{\text {II }}\right] 6548 \AA=3.0$.

the approaching jet in all observations. The blue-shifted side is also extended (see Fig. 5), but since the jet is seen on this side we cannot distinguish whether this emission is entirely due to the jet or if there is an additional component similar to the one shown in Fig. 7. In contrast, the Si III line is consistent with the continuum, which is not spatially extended.

The two observations taken in 2000 are less than a day apart, and the spatial profiles have a very similar shape. Thus, it is unlikely that the difference in flux between them is due to temporal variability. Instead, it is caused by the aperture. One observation used a slit width of $0.2^{\prime \prime}$, the other $0.5^{\prime \prime}$. Both spatial profiles are very similar, so the bulk of the emission in the direction perpendicular to the slit and thus also perpendicular to the jet, must be contained within $0.2^{\prime \prime}$.

We searched for a similar feature in the Si III line at $1206.51 \AA$, but the projections of line and background are fully compatible here.

\subsection{Optical emission}

PVDs of [O I] $6300 \AA, \mathrm{H} \alpha$, and the [S II] lines at $6716 \AA$ and $6731 \AA$ are shown in Fig. 5 (right). Stray light is strongly reduced due to the occulting wedge used in the observations, but the inner few arcseconds of the long-slit data are contaminated by Airy 

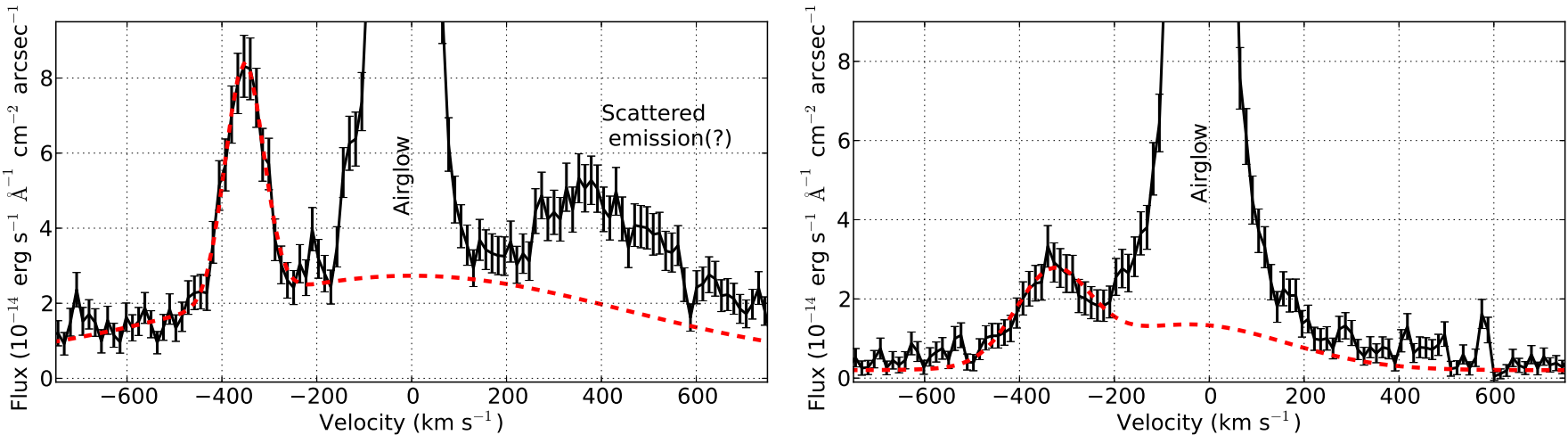

Fig. 6. Spectra of the Ly $\alpha$ line in the two brightest knots. The inner part of the line is contaminated by airglow. Additionally, the knot closer to the source (left panel) shows some emission on the red side of the line which could be due to scattered light from the central star (see text). We fit two Gaussians for knot and background (dashed line). Both knots appear blue-shifted by about $350 \mathrm{~km} \mathrm{~s}^{-1}$.

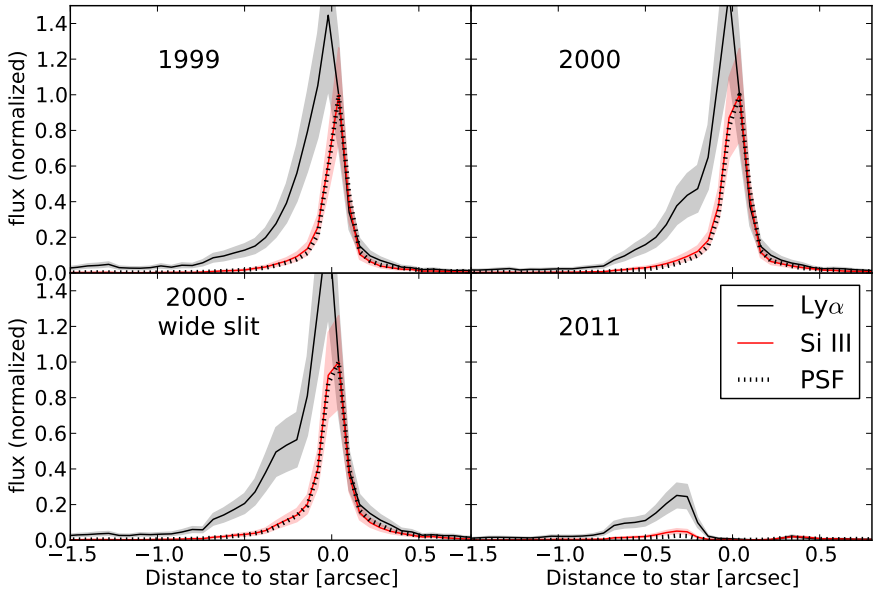

Fig. 7. Spatial profile on the red ( 250 to $450 \mathrm{~km} \mathrm{~s}^{-1}$ ) side of the Ly $\alpha$ line, the Si III line, and of a continuum region. All line profiles and continuum profiles are normalized (see text for details). Shaded regions indicate the statistical uncertainty for the lines. The uncertainty on the continuum profile is negligible because it is extracted from a much larger region.

rings caused by refraction of the bright central star. We fit and remove the Airy rings, but the residual image shows a higher noise level at these positions.

At first sight, the $\mathrm{H} \alpha$ emission seems extended in the direction of the blue-shifted jet - not only for the jet velocity, but also for a wide range from -300 to $+300 \mathrm{~km} \mathrm{~s}^{-1}$. A projection similar to Fig. 7 shows that this apparent extension is indistinguishable from the spatial profile of the continuum. The only reason it shows up prominently here is that the $\mathrm{H} \alpha$ line is far brighter than the stellar continuum.

Two knots can be identified in the counterjet, but no feature is seen in the jet itself (Table 3). The strongest knot (which we call B2) peaks at $3.3^{\prime \prime}$, very close to the outer edge of the disk as seen in scattered light (Grady et al. 2000). Even without contamination by the Airy rings, the disk would block any emission from the counterjet closer in, thus we cannot say if the observed position of B2 represents the peak of the knot or if we merely observe the end of a more extended structure. The second knot (B) is located at 8.7". B2 and B show a similar spacing to B and C in the old observation (Wassell et al. 2006).

Using the extraction regions 2.6-3.7" and 8.1-9.0" we extract spectra for B2 and B, respectively (Fig. 8). In addition to

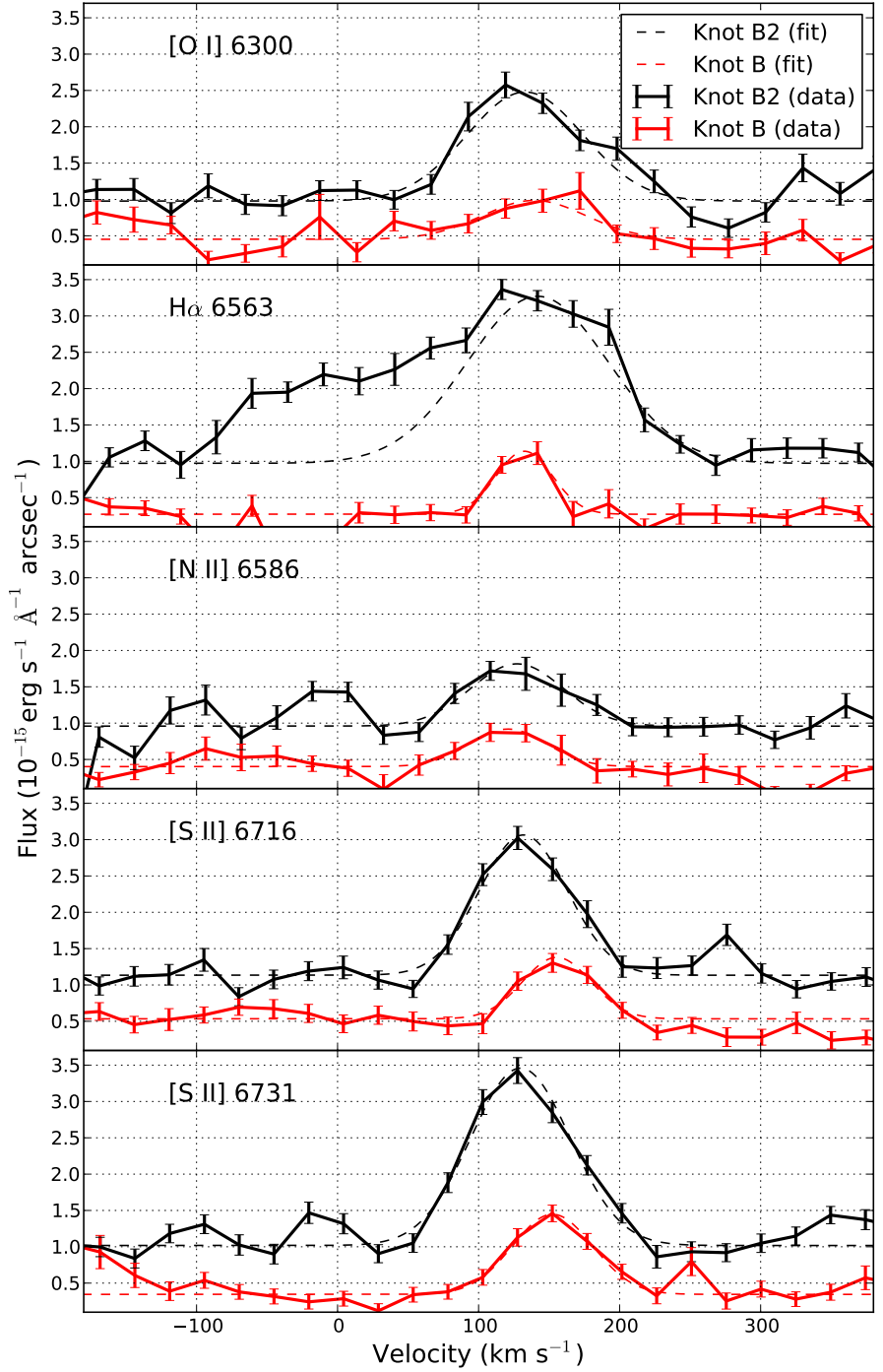

Fig. 8. Spectra for $\mathrm{H} \alpha$ and several optical FELs at the position of the two brightest knots. The main component is fitted with a Gaussian.

the lines shown in the PVD we include [N II] $6586 \AA$ A. Gaussian fits to the lines are given in Table 4, and $\mathrm{H} \alpha$ is described by two Gaussians. The table reports the component with a higher velocity shift, since the inner component is most likely due to 
scattered stellar emission. Owing to the lower spectral resolution $\mathrm{H} \alpha$ and $\left[\mathrm{N}_{\mathrm{II}}\right]$, and the two [S $\mathrm{SI}_{\mathrm{II}}$ lines at 6716 and $6731 \AA$ were blended in the previous optical STIS spectra. We remeasured the line fluxes from 1999, but our values are consistent with Wassell et al. (2006) within the errors.

The line fluxes in the table are not dereddened. The wavelength range of the optical observations is narrow, so a very similar correction applies to all those lines. The same $E(B-V)=0.015$ as above leads to a flux correction of $3-4 \%$ in the optical. This is much less than the statistical errors on the line fluxes.

In knot B2 in the counterjet the velocities of all lines are roughly compatible with a systematic knot velocity of $130 \mathrm{~km} \mathrm{~s}^{-1}$. The forbidden lines all have a similar width with only the $\mathrm{H} \alpha$ line significantly wider (Table 4). In knot B the $\left[\mathrm{S}_{\mathrm{II}}\right]$ lines seem slightly more red-shifted than the other observed lines, however the errors given in the table are only the statistical uncertainty. In general, the velocities in the optical are lower and narrower than in the $\operatorname{Ly} \alpha$ line. Wassell et al. (2006) have already found a velocity asymmetry between jet and counterjet but at much lower level and with larger errors $(223 \pm 81$ vs. $171 \pm 89 \mathrm{~km} \mathrm{~s}^{-1}$ ). Apparently, the Ly $\alpha$ emission in the jet traces a faster component than the FELs. In our observation the FUV features in the jet are not detected in the optical, thus a comparison of FUV and optical lines in the same knot is not possible. Velocity asymmetries are usual. The ratio of jet velocity and counterjet velocity is $>1.5$ for about half of all CTTS with optically detected outflows. Those differences seem to be intrinsic to the launching process and cannot be explained by interactions with the circumstellar matter close to the central source (Hirth et al. 1994).

\subsubsection{Proper motion and velocity of knot $B$}

Knot B was seen at $4.3^{\prime \prime}$ in 1999 . For reasonable values of its proper motion, it should be located a few arcsec farther out in the new STIS observation. Indeed, a knot is located at 8.7", and we identify this as knot B with a proper motion of $0.37 \pm 0.01^{\prime \prime} \mathrm{yr}^{-1}$, which agrees well with the proper motion of knot $C$ of $(0.34 \pm$ $0.02)^{\prime \prime} \mathrm{yr}^{-1}$. Thus, it seems that knots in the jet move consistently with similar velocities.

We take the velocity along the line of sight as the mean of all observed line shifts for knot B (Table 4) and derive a total space motion of $250 \mathrm{~km} \mathrm{~s}^{-1}$ for knot $\mathrm{B}$ and an inclination of $56^{\circ}$. The proper motion measures the motion of the shock front, while the line shift shows the motion of the gas. Thus, the above calculation requires that the shock front is comoving with the material. As shown below, the relative shock speed is low, so this approximation is valid. The measured values agree with previous measurements of the jet speed and inclination.

\subsubsection{Physical conditions in knot B}

While the observed surface luminosity in knot B2 is about two to three times higher than in knot B, the line ratios are very similar, which indicates a similar density and temperature, although knot B is nearly twice as far from the central source as knot B2 (integrated fluxes are given in Table 4). A decade ago, when knot B was located close to the current position of knot B2, its surface luminosity in $\mathrm{H} \alpha$ and [S II] was significantly higher than knot B today. The surface luminosity of knot B in the [S II] and [O I] lines reduced by a factor of 2 , in [N II] by a factor of 4 , but in $\mathrm{H} \alpha$ by an order of magnitude between 1999 and 2011.
Table 5. Ionization fraction and $v_{\text {shock}}$.

\begin{tabular}{lccc}
\hline \hline Line ratio & $\mathrm{B}(1999)$ & $\mathrm{B}(2011)$ & $\mathrm{B} 2(2011)$ \\
\hline \multicolumn{4}{c}{ Ionization fraction } \\
{$\left[\mathrm{O}_{\mathrm{I}}\right] / \mathrm{H} \alpha$} & 0.2 & 0.02 & 0.03 \\
{$\left[\mathrm{~S}_{\mathrm{II}}\right](6716+6731) / \mathrm{H} \alpha$} & 0.07 & 0.02 & 0.04 \\
{$\left[\mathrm{~N}_{\mathrm{II}}\right] /\left[\mathrm{O}_{\mathrm{I}}\right]$} & 0.3 & 0.1 & 0.1 \\
\hline \multicolumn{5}{c}{ Shock velocity $\left[\mathrm{km} \mathrm{s}^{-1}\right]$} \\
{$\left[\mathrm{O}_{\mathrm{I}}\right] / \mathrm{H} \alpha$} & 50 & 30 & 40 \\
{$\left[\mathrm{~S}_{\mathrm{II}}\right](6716+6731) / \mathrm{H} \alpha$} & 40 & 30 & 30 \\
{$\left[\mathrm{~N}_{\mathrm{II}}\right] /\left[\mathrm{O}_{\mathrm{I}}\right]$} & 70 & 30 & 30 \\
{$\left[\mathrm{~S}_{\mathrm{II}}\right] 6716 /\left[\mathrm{S}_{\mathrm{II}}\right] 6731$} & $\ldots$ & 45 & 40 \\
\hline
\end{tabular}

\subsubsection{Electron densities}

The ratio of the [S $\left.\mathrm{S}_{\mathrm{II}}\right]$ lines at $6716 \AA$ and $6731 \AA$ is sensitive to density with only a very modest temperature dependence. The differential reddening between those two lines is negligible, because HH 409 is only weakly reddened and the wavelength is very similar. Unfortunately, these lines are unresolved in the early STIS observations, so that we do not have any comparison value for knot B from an earlier epoch. However, in 2004 the density of knot $\mathrm{C}$ was measured from ground-based data to $n_{\mathrm{e}}=1400 \pm 400 \mathrm{~cm}^{-3}$ (Wassell et al. 2006). With the observed line values from Table 4 we find $n_{\mathrm{e}}=2200_{-600}^{+1000} \mathrm{~cm}^{-3}$ and $n_{\mathrm{e}}=3000_{-1000}^{+3000} \mathrm{~cm}^{-3}$, for knots $\mathrm{B} 2$ and $\mathrm{B}$, respectively, using the CHIANTI database (Dere et al. 1997; Landi et al. 2012). Below we calculate the total density $n$ from $n_{\mathrm{e}}$ and the ionization fraction.

\subsubsection{Shock velocities}

We estimate the shock velocities and the ionization fraction in the knots using the models of Hartigan et al. (1994). They contain updates for some optical lines over the simulations by Hartigan et al. (1987), which we used to interpret the Ly $\alpha / \mathrm{Si}$ III ratio. Hartigan et al. (1994) present a series of plots for different line ratios that provide a diagnostic of the ionization fraction and the shock velocity. The models were calculated for three different pre-shock densities and for different magnetic fields. Given the estimate of the electron density above, we use the models with a pre-shock density of $10^{3} \mathrm{~cm}^{-3}$. The magnetic field in the shock zone is degenerate with the shock speed because a field parallel to the shock front inhibits strong compression. We assume a weak field $(<0.1 \mathrm{mG})$ here.

Table 5 shows the ionization fractions $\langle I\rangle$ (averaged over the emitting area) and the shock velocities $v_{\text {shock }}$ from the Hartigan et al. (1994) models. The shock velocities derived from different line ratios agree well. Knots B and B2 in the most recent observation have a very similar shock speed around $30 \mathrm{~km} \mathrm{~s}^{-1}$, less than the shock speed of knot B in 1999. The differences between the ionization fractions are much larger. Again, the knot B in 1999 was more highly ionized than any knot in 2011; however, the recent values span a range of a factor of five. This could be due to underestimating the $\mathrm{H} \alpha$ flux in the fitting process. In knot B2 the fitted Gaussian has a higher velocity than all other lines. Thus, a larger fraction of the flux is described by the Gaussian of a stationary component, which we interpret as scattered light from the central source. On the other hand, the relative errors on the line fluxes for weak lines are large and the differences in $\langle I\rangle$ in Table 5 are only marginally significant. In the following we adopt a shock velocity of $50 \mathrm{~km} \mathrm{~s}^{-1}$ for knot B in 1999 and $35 \mathrm{~km} \mathrm{~s}^{-1}$ for knots B and B2 in 2011. The shock 
models predict a compression $\langle C\rangle$ of gas (averaged over the [S II] emitting area) of a factor 50 and 25 for these shock velocities.

\subsubsection{Mass loss rates}

There are several different methods of estimating the mass flux in a jet. In this section we follow Hartigan et al. (1994). First, the mass flux can be estimated from the total flux in a line, e.g. [O I]. We calculate the total luminosity and assume that the plasma is emitting close to the peak formation temperature of this line and that the total oxygen abundance is close to solar. This calculation misses flux components that are located outside the aperture, ionized, too cool to emit [O I] $6300 \AA$, or that have densities above the critical density for [O I], e.g. in a dense and fast component very close to the jet axis. While we know little about temperature components outside of the formation range of FELs, the density is most likely below the critical density $\left(10^{6} \mathrm{~cm}^{-3}\right)$ given the estimates above. We use

$$
\begin{aligned}
\dot{M}= & 5.95 \times 10^{-8}\left(\frac{n_{\mathrm{e}}}{10^{3} \mathrm{~cm}^{-3}}\right)^{-1}\left(\frac{L_{6300}}{10^{-4} L_{\odot}}\right) \\
& \times\left(\frac{v_{\text {sky }}}{100 \mathrm{~km} \mathrm{~s}^{-1}}\right)\left(\frac{l_{\text {sky }}}{10^{16} \mathrm{~cm}}\right)^{-1} M_{\odot} \mathrm{yr}^{-1},
\end{aligned}
$$

which is Eq. (10) from Hartigan et al. (1994). Alternatively, a very similar calculation can be done using the $\left[\mathrm{S}_{\mathrm{II}}\right]$ lines. If they are within the high-density limit $\left(>10^{4} \mathrm{~cm}^{-3}\right)$, then the formula does not depend on the density in the knot (Hartigan et al. 1995, Eq. (A.10)):

$$
\begin{aligned}
\dot{M}= & 4.5 \times 10^{-9}\left(\frac{L_{6731}}{10^{-4} L_{\odot}}\right)\left(\frac{v_{\text {sky }}}{100 \mathrm{~km} \mathrm{~s}^{-1}}\right) \\
& \times\left(\frac{l_{\text {sky }}}{10^{16} \mathrm{~cm}}\right)^{-1} M_{\odot} \mathrm{yr}^{-1} .
\end{aligned}
$$

Finally, we can calculate the mass flux through an area A, when the gas moves at a velocity $v$ :

$\dot{M}=\rho v \mathrm{~A}=\mu m_{\mathrm{H}} n v \mathrm{~A}$,

where $\rho=\mu m_{\mathrm{H}} n$ is the mass density, calculated from the mean molecular weight $\mu=1.24$, the mass of the hydrogen atom $m_{\mathrm{H}}$, and the atom/ion number density $n$. The difficulty here is to estimate $n$ from the measured electron density $n_{\mathrm{e}}$. This can be done using ionization $\langle I\rangle$ and the compression $\langle C\rangle$ from the shock models of Hartigan et al. (1994). These authors recommend scaling the density with $\sqrt{\langle C\rangle}$ because this is more appropriate for a clumpy medium than scaling with the compression factor itself. We get

$n=\frac{n_{\mathrm{e}}}{\sqrt{\langle C\rangle}\langle I\rangle}$.

Since we do not measure the size of the jet perpendicular to the direction of the flow, we use a radius of $0.15^{\prime \prime}$, which is the width of the innermost knots resolved by Wassell et al. (2006). We take the total space motion of knot B as the flow speed of material. Table 6 gives the mass flux in knots B and B2 estimated from all three methods. Given the simplifications, the difference in the mass flux measured is not surprising. The densities of knots B and B2 are very similar, but their luminosities differ. This indicates that we see a larger emitting volume in knot B2. In knot B the mass flux calculated from the flow is much larger than the mass flux from the line luminosities. This could indicate that either a larger part of knot is too cool to radiate in [O I] or [S II] or that the knot has a small filling factor; i.e., part of the volume contains cool material unrelated to the jet (Podio et al. 2006).
Table 6. Mass flux in the knots.

\begin{tabular}{lccc}
\hline \hline & $\mathrm{B}(1999)$ & $\mathrm{B}(2011)$ & $\mathrm{B} 2(2011)$ \\
\hline$n_{\mathrm{e}}\left[\mathrm{cm}^{-3}\right]$ & $\approx 3000$ & 3000 & 2200 \\
$\langle C\rangle$ & 50 & 25 & 25 \\
$\langle I\rangle$ & 0.2 & 0.05 & 0.05 \\
$l_{\text {sky }}\left[10^{14} \mathrm{~cm}\right]$ & 6 & 6 & 6 \\
$v_{\text {sky }}\left[\mathrm{km} \mathrm{s}^{-1}\right]$ & 190 & 190 & 210 \\
$L_{6300}\left[10^{-7} L_{\odot}\right]$ & 2 & 1 & 3 \\
$L_{6731}\left[10^{-7} L_{\odot}\right]$ & $\ldots$ & 2 & 5 \\
$n\left[\mathrm{~cm}^{-3}\right]$ & $\ldots$ & $12 \times 10^{3}$ & $9 \times 10^{3}$ \\
$\dot{M}_{6300}\left[10^{-9} M_{\odot} \mathrm{yr}^{-1}\right]$ & 1.3 & 0.6 & 2.8 \\
$\dot{M}_{6731}\left[10^{-9} M_{\odot} \mathrm{yr}^{-1}\right]$ & $\ldots$ & 0.3 & 0.8 \\
$\dot{M}_{\text {flow }}\left[10^{-9} M_{\odot} \mathrm{yr}^{-1}\right]$ & $\ldots$ & 2.8 & 2.2 \\
\hline
\end{tabular}

\section{Discussion}

\subsection{The central star}

HD 163296 is remarkably constant over nearly 200 ks of X-ray observing time. This is consistent with the abundance pattern of an inactive star and the cool temperatures. In contrast, CTTS are typically active stars with frequent flaring and an inverse FIP abundance pattern. A jet collimation shock could explain some of the soft emission. However, no offset of the X-ray emission from the central star is seen, and we estimate an upper limit of $30 \mathrm{AU}$. This is stricter than the number given in Günther \& Schmitt (2009), and we can thus calculate a more stringent limit on the conditions at the jet base, assuming that a shock at the jet base is the origin of the central soft X-ray component. The post-shock cooling length $d_{\text {cool }}$ only depends on the pre-shock density $n_{0}$ and shock temperature and can be written as (Raga et al. 2002):

$d_{\text {cool }} \approx 8 \mathrm{AU}\left(\frac{10^{5} \mathrm{~cm}^{-3}}{n_{0}}\right)\left(\frac{v_{\text {shock }}}{400 \mathrm{~km} \mathrm{~s}^{-1}}\right)^{4.5}$.

Günther \& Schmitt (2009) derived $v_{\text {shock }}=400 \mathrm{~km} \mathrm{~s}^{-1}$, thus the lower limit on the density is $n_{0}>2 \times 10^{4} \mathrm{~cm}^{-3}$. The flow time through this post-shock region would be about a year. In turn, the density sets an upper limit on the radius $R$ of the shock front assuming a circular shock at the base of a cylinder (for details see Günther et al. 2009)

$R \approx 1.6\left(\frac{10^{5} \mathrm{~cm}^{-3}}{n_{0}}\right)^{0.5}\left(\frac{V E M}{10^{52} \mathrm{~cm}^{-3}}\right)^{0.5}\left(\frac{0.21 \mathrm{keV}}{\mathrm{k} T}\right)^{1.125}$

where $R$ is given in AU. With the VEM from Table 2 this leads to an upper limit $R<5 \mathrm{AU}$. This radius would require a rather large opening angle at the base of the jet, thus it is likely that the actual densities are higher than the lower limit derived here. The mass flux required to power the X-ray emission is $6 \times 10^{-11} M_{\odot} \mathrm{yr}^{-1}$, only about a tenth of the mass flux in the outer optical knots (Table 6). Thus, this seems to be powered by a different (faster and less massive) component of the outflow than the optical knots in the jet.

\subsection{The one-sided Ly $\alpha$ emission}

We find a significant extension of the Ly $\alpha$ line in the direction of the approaching jet in all observations. The signal on the blueshifted side of the emission line could partly be caused by the jet itself, but the red-shifted emission has to come from a different origin. The emission is seen between $0.1^{\prime \prime}$ and $1^{\prime \prime}$, which corresponds to 12 and $120 \mathrm{AU}$ if the emitting structure is in the 
plane of the sky. Perpendicular to the jet the emission is mostly contained in the inner $0.2^{\prime \prime}$. The emission reaches as far out as $400 \mathrm{~km} \mathrm{~s}^{-1}$ as seen, e.g., at the position of the first knot (see Fig. 6, where this feature is labeled "scattered emission?"), but any signal within $150 \mathrm{~km} \mathrm{~s}^{-1}$ of the line center is hidden behind interstellar absorption and geocoronal emission.

This feature has already been noticed by Devine et al. (2000) who suggest that it is caused by infall or a poorly collimated wind. We disagree with this explanation because of the large width of the observed line. Thermal broadening cannot produce the width since hydrogen would be fully ionized at the required temperatures and pressure broadening only becomes relevant for densities on the order of $10^{13} \mathrm{~cm}^{-3}$ (Vidal et al. 1973). Thus, the line width must be explained by kinematic motions but at distances of a few AU where the feature is seen the gravity of the central star provides an accretion flow speed of only a few $\mathrm{km} \mathrm{s}^{-1}$, much less than the width of the emission line.

The disk obscures the far side of the system, so that we only see the approaching wind. If we were looking pole-on, all outflows on the visible side would be blue-shifted, but since the inclination is $50-60^{\circ}$ some outflows might appear red-shifted. In the extreme case of a flat disk and a disk wind moving in parallel to the disk, the flow speed still has to reach $500 \mathrm{~km} \mathrm{~s}^{-1}$ in order to achieve a line-of-sight velocity of $400 \mathrm{~km} \mathrm{~s}^{-1}$. Not only is the limiting case of a flat disk unrealistic, the required velocity for this outflow is also much higher than the jet velocity. Although we do not understand the jet collimation process in detail, current models suggest that the collimation happens within a few stellar radii (e.g. Lii et al. 2012), and commonly the less-collimated layers of the outflow are much slower. One mechanism for such an outflow is a photo-evaporative disk wind, where the upper layer of the disk is heated by the stellar radiation. This layer is hot enough to emit in $\operatorname{Ly} \alpha$, but predicted velocities for CTTS are only a few $\mathrm{km} \mathrm{s}^{-1}$ (Ercolano \& Owen 2010; Pascucci et al. 2011). Even for HAeBe, which are much brighter in the UV, these winds will not reach the required velocities. Additionally, all the above models would predict an equally strong blue-shifted emission, which is not observed.

These difficulties make it promising to propose a model where the $\operatorname{Ly} \alpha$ flux seen at some distance to the central source does not originate there but is instead scattered emission from the central source, since a spectrum extracted at the stellar position shows that the Ly $\alpha$ line is wide enough. Presumably, most of the hydrogen emission originates in the accretion funnel and the accretion shock (Muzerolle et al. 1998). However, dust scattering is not wavelength-selective and would thus lead to comparable spatial profiles for the FUV continuum and the wings of the Ly $\alpha$ line in contrast to Fig. 7. (The same argument makes an instrumental artifact unlikely). This can be overcome if the Ly $\alpha$ line is formed in the accretion funnels above the plane of the disk. The stellar continuum would be absorbed by the inner disk wall, while the accretion funnels could reach above the rim and illuminate the disk surface out to the required radius. Wisniewski et al. (2008) observe dark lanes in scattered optical light a few arcseconds from the central source, which correlate with the overall disk luminosity and are consistent with shadowing by a time-variable inner disk wall. An alternative explanation is a dusty disk wind, with dust grains entrained in the outflow (Bans \& Königl 2012). A flared disk or a dusty disk wind would intercept a large fraction of the total $\operatorname{Ly} \alpha$ flux. In principle, we should see Ly $\alpha$ from the entire disk surface but it is only observed in the negative spatial direction (below the star in Fig. 5 and to the left in Fig. 7). This is the far side of the disk that we see through the jet, so the light needs to be back-scattered to reach us. However, dust is strongly forward scattering in the UV (Draine 2003), and thus the strongest signal should be observed in the positive spatial direction. The scattering angles in a dusty wind are slightly more favorable than in a flared disk.

Alternatively, scattering by dust in the jet would explain naturally why we see the extended emission on the side of the jet only and also why it does not seem to be extended perpendicular to the jet axis. Also, the required scattering angle is the inclination of the jet (about $50^{\circ}$ ), which is in the preferred range of angles. A potential problem with this scenario is that there is no direct observational evidence for dust in the jet. Theoretically, if the jet material is launched close enough to the central star, all dust may be destroyed near the jet base, by stellar irradiation and/or thermal evaporation, although detailed calculations are needed to quantify the dust content of the jet.

Since the spatial profiles from observations between 1999 and 2011 - 12 years apart - are compatible, it is unlikely that a feature in the disk; e.g., a density enhancement on the far side of the disk causes the scattering to be much more efficient on one side than on the other.

We conclude that several components of dust scattering, wind emission, and wind absorption with different speeds and launching radii need to be present at the same time to produce the observed signature.

\subsection{Time evolution of the knots}

Knot B is the only knot observed in 1999 and in 2011. In Sect. 4.3.1 we have already derived its proper motion. In this time period the observed surface luminosity decreased significantly. This could be due to intrinsic dimming or could indicate that the jet width increased beyond the width of the slit used in the observations. The lowest difference - a factor $2-$ is observed for [O I], and the largest difference - a factor 10 - is observed for $\mathrm{H} \alpha$. Consequently, the ionization fraction and shock speed deduced from the line ratios are lower in 2011 than in 1999.

Except for the $\left[\mathrm{S}_{\mathrm{II}}\right]$ doublet, no element is observed with more than one line, thus the temperature of the emitting material cannot be measured independently of the abundances. However, since all observed elements are neutral or singly ionized, plasma with temperatures between 5000 and $25000 \mathrm{~K}$ must be present. In this energy range, atomic lines are the dominant contributors to line cooling. As an estimate, we use the radiative loss rate $\Lambda=5 \times 10^{-23} \mathrm{erg} \mathrm{cm}^{3} \mathrm{~s}^{-1}$ which is valid for this temperature range within a factor of a few. The cooling time $\tau$ can be calculated as the ratio of the thermal energy content of the gas and the radiative loss as

$\tau=\frac{3 / 2 n k T}{\Lambda n n_{\mathrm{e}}} \approx 0.4 \mathrm{yr}$

where $k$ is the Boltzmann constant and we use the $n_{\mathrm{e}}$ and $n$ estimated in Sect. 4.3.3. The short time scale shows that the knot must be continuously reheated by a shock that converts the kinetic energy of the jet into thermal energy. Ultimately, the radiation is thus powered from the kinetic energy of the jet. The kinetic energy density of the material is 300 times higher than the thermal energy density. Indeed, $v_{\text {sky }}$ and the shock velocity are both lower in the 2011. This is consistent with a jet that decelerates because its kinetic energy is radiated away. An additional consequence is that the measured proper motion, which is determined from the position of the shock front, is not the same as the proper motion of the mass in the jet.

The knots $\mathrm{A}$ and $\mathrm{C}$ are not visible in the new observation. As shown above the cooling times for gas in the knots are very 
short, so no detection is expected, if the shock fronts in these knots are no longer strong enough to power the radiation.

Due to the low signal in the X-ray observations we cannot derive meaningful limits on the density and cooling time, but we note that in DG Tau the outer X-ray knots are less luminous than the inner ones (Güdel et al. 2008), and a cooling of the X-ray plasma as it travels outward has been observed by Schneider et al. (2011).

\subsection{Long-term evolution of the jet}

In ground-based observations Wassell et al. (2006) are able to identify fainter knots farther away from the driving source, and they estimate a dynamical age of HH 409 of at least $80 \mathrm{yr}$. Our observations are not as sensitive to fainter structures, but in the inner jet we can confirm that the general shape of the jet has not changed over one decade. We have discovered a new knot (B2), which has a similar velocity as the previously innermost knot B. The distance to the preceding knot is also comparable, indicating a periodicity for knot launching; however, knot B2 has a lower shock velocity and compression factor, but slightly higher electron density and mass loss rate than knot B had at a comparable distance from the driving source. This shows that the launching conditions must have changed slightly.

\subsection{Implications for jet launching}

Most theoretical models rely on magnetic fields to explain the jet launching and collimation. Nevertheless, it is unclear if the central star HD 163296 possesses a large-scale magnetic field. On the one hand, none was found in the observations of Hubrig et al. (2007); on the other hand, we observe a constant X-ray flux with temperatures above $2 \mathrm{keV}$, which is beyond the heating from an accretion shock and is presumably caused by magnetic reconnection and thus indicates the consistent presence of stellar magnetic fields.

We know that knot B2 was launched within a few years of the optical polarimitry and the X-ray observations. If it has moved with constant proper motion, then it was launched in 2002, while the observations of Hubrig et al. (2007) were performed in 2004, and we analyzed X-ray data from 2003, 2007, and 2011. If the limit on the magnetic field is representative, then a strong stellar magnetic field is not a necessary prerequisite of jet launching. Instead, Hubrig et al. (2007) report detecting Stokes components in the $\mathrm{Ca} \mathrm{H}$ and $\mathrm{K}$ lines, which are much wider than expected from the photosphere. If these lines are of circumstellar origin, they probe the jet launching and collimation region more directly. These fields could be generated in, e.g., a disk dynamo (Vishniac \& Brandenburg 1997).

Alternatively, we can take the presence of hard X-rays as proof of stellar magnetic fields. The optical polarimitry concentrated on hydrogen lines, which - at least in CTTS - have multiple contributions from the inner edge of the disk, the accretion funnel and the star. The stellar contribution of the flux might be too small to produce a measurable Stokes signal. Thus, we argue that the jet launching and collimation in HAeBes is comparable to the process in CTTS.

\section{Summary}

We analyze a series of Chandra and HST observations of HH 409, the jet from the Herbig Ae star HD 163296. In X-rays the star itself is very stable, and the observed temperatures and emission measures are fully compatible with previous observations. No extension beyond the PSF was found for the central star, which limits any unresolved sources with a significant fraction of the total luminosity to a maximum separation of $30 \mathrm{AU}$. This is important because an XMM-Newton spectrum showed that the soft X-ray emission originates above the stellar surface, possibly in the jet collimation region. While in 2003 a weak feature on the edge of the stellar PSF was observed at a distance of -4 ", it is unclear whether emission at that position is present in 2011. Swartz et al. (2005) claim to detect a knot in the jet at $-7.2^{\prime \prime}$; our analysis confirmed this. There is a tentative grouping of three photons at $-10.6^{\prime \prime}$ in 2011 . This position is consistent with the $2003 \mathrm{knot}$, if it propagates with the same proper motion as the optical knots. In any case, the luminosity of the knot has dropped significantly since 2003, indicating a short cooling time.

In the UV the Ly $\alpha$ line is the most prominent feature. In the new observations, we detect two knots in the jet, but not the counterjet. In addition, there is extended emission on the side of the approaching jet that is red-shifted up to $400 \mathrm{~km} \mathrm{~s}^{-1}$ and can be seen out to about $1^{\prime \prime}$. On the blue-shifted side, the emission is much weaker. Neither red nor blue-shifted emission is seen in the direction of the counterjet. Multiple components of accretion, a wide-angle wind, or dust scattering of the Ly $\alpha$ line from the accretion spot are required to explain this feature.

In the optical we detected $\mathrm{H} \alpha$ and several forbidden emission lines. We detected a new knot, called B2, and re-observed the previously detected knot B. The distance between those two knots is similar to the distance between knot B and the preceding knot. This fits nicely into a series of knots in the counterjet with almost equal spacing, which were launched over several decades (Wassell et al. 2006). Knot B moves with a proper motion compatible to the previous values for knot C. Knot B is significantly fainter now than in 1999, particularly in $\mathrm{H} \alpha$. The line ratios indicate a lower temperature. A significant spatial expansion, which would bring the size of the knot beyond the width of the slit, could also contribute to the fading. Still, the cooling time scale is less than a year, so knot B needs to be continuously reheated. This supports a picture where the knots are shock fronts traveling though a jet of continuously launched, cool material. Over time, these shocks radiate away their energy, so the post-shock cooling zone formed behind them has consecutively lower temperatures.

Knot B2 today is seen at almost the same distance from the central star as knot B was a decade ago. Nevertheless, knot B2 has lower ionization and radial velocity, but a larger mass flux than knot B had a decade ago. Thus, the jet launching conditions must have changed in this period.

Acknowledgements. Support for this work was provided by the National Aeronautics and Space Administration (NASA) through Chandra Awards Number GO2-13015X and TM0-11002X issued by the Chandra X-ray Observatory Center, which is operated by the Smithsonian Astrophysical Observatory for and on behalf of NASA under contract NAS8-03060. Further support comes from grant GO-12186.01-A from the Space Telescope Science Institute, which is operated by the Association of Universities for Research in Astronomy, Inc., under NASA contract NAS5-26555. P.C.S. acknowledges support from the DLR under 50 OR 1112. Some of the data presented in this paper were obtained from the Mikulski Archive for Space Telescopes (MAST).

\section{References}

Anderson, J. M., Li, Z.-Y., Krasnopolsky, R., \& Blandford, R. D. 2005, ApJ, 630, 945

Bacciotti, F., Mundt, R., Ray, T. P., et al. 2000, ApJ, 537, L49

Bally, J., Feigelson, E., \& Reipurth, B. 2003, ApJ, 584, 843

Bans, A., \& Königl, A. 2012, ApJ, 758, 100

Blandford, R. D., \& Payne, D. G. 1982, MNRAS, 199, 883

Cabrit, S., Edwards, S., Strom, S. E., \& Strom, K. M. 1990, ApJ, 354, 687 
Carter, C., Karovska, M., Jerius, D., Glotfelty, K., \& Beikman, S. 2003, in Astronomical Data Analysis Software and Systems XII, eds. H. E. Payne, R. I. Jedrzejewski, \& R. N. Hook, ASP Conf. Ser., 295, 477

Coffey, D., Bacciotti, F., \& Podio, L. 2008, ApJ, 689, 1112

Dere, K. P., Landi, E., Mason, H. E., Monsignori Fossi, B. C., \& Young, P. R. 1997, A\&AS, 125, 149

Devine, D., Grady, C. A., Kimble, R. A., et al. 2000, ApJ, 542, L115

Doe, S., Nguyen, D., Stawarz, C., et al. 2007, in Astronomical Data Analysis Software and Systems XVI, eds. R. A. Shaw, F. Hill, \& D. J. Bell, ASP Conf. Ser., 376, 543

Draine, B. T. 2003, ApJ, 598, 1017

Eislöffel, J., \& Mundt, R. 1998, AJ, 115, 1554

Ercolano, B., \& Owen, J. E. 2010, MNRAS, 406, 1553

Favata, F., Bonito, R., Micela, G., et al. 2006, A\&A, 450, L17

Fruscione, A., McDowell, J. C., Allen, G. E., et al. 2006, in SPIE Conf. Ser., 6270

Garcia Lopez, R., Natta, A., Testi, L., \& Habart, E. 2006, A\&A, 459, 837

Grady, C. A., Devine, D., Woodgate, B., et al. 2000, ApJ, 544, 895

Grevesse, N., \& Sauval, A. J. 1998, Space Sci. Rev., 85, 161

Güdel, M., Skinner, S. L., Briggs, K. R., et al. 2005, ApJ, 626, L53

Güdel, M., Skinner, S. L., Audard, M., Briggs, K. R., \& Cabrit, S. 2008, A\&A, 478, 797

Güdel, M., Audard, M., Bacciotti, F., et al. 2011, in 16th Cambridge Workshop on Cool Stars, Stellar Systems, and the Sun, eds. M. Christopher, M. Johns-Krull, M. K. Browning, \& A. A. West (San Francisco: ASP), ASP Conf. Ser., 448, 617

Günther, H. M., \& Schmitt, J. H. M. M. 2009, A\&A, 494, 1041

Günther, H. M., Matt, S. P., \& Li, Z.-Y. 2009, A\&A, 493, 579

Hartigan, P., Raymond, J., \& Hartmann, L. 1987, ApJ, 316, 323

Hartigan, P., Morse, J. A., \& Raymond, J. 1994, ApJ, 436, 125

Hartigan, P., Edwards, S., \& Ghandour, L. 1995, ApJ, 452, 736

Hirth, G. A., Mundt, R., Solf, J., \& Ray, T. P. 1994, ApJ, 427, L99

Hubrig, S., Pogodin, M. A., Yudin, R. V., Schöller, M., \& Schnerr, R. S. 2007, A\&A, 463, 1039
Juda, M., \& Karovska, M. 2010, in AAS/High Energy Astrophysics Division \#11, BAAS, 42, 722

Kwan, J., \& Tademaru, E. 1988, ApJ, 332, L41

Landi, E., Del Zanna, G., Young, P. R., Dere, K. P., \& Mason, H. E. 2012, ApJ, 744, 99

Li, J., Kastner, J. H., Prigozhin, G. Y., et al. 2004, ApJ, 610, 1204

Lii, P., Romanova, M., \& Lovelace, R. 2012, MNRAS, 420, 2020

Matt, S., \& Pudritz, R. E. 2005, ApJ, 632, L135

Montesinos, B., Eiroa, C., Mora, A., \& Merín, B. 2009, A\&A, 495, 901

Muzerolle, J., Calvet, N., \& Hartmann, L. 1998, ApJ, 492, 743

Pascucci, I., Sterzik, M., Alexander, R. D., et al. 2011, ApJ, 736, 13

Podio, L., Bacciotti, F., Nisini, B., et al. 2006, A\&A, 456, 189

Pravdo, S. H., Feigelson, E. D., Garmire, G., et al. 2001, Nature, 413, 708

Raga, A. C., Noriega-Crespo, A., \& Velázquez, P. F. 2002, ApJ, 576, L149

Schneider, P. C., \& Schmitt, J. H. M. M. 2008, A\&A, 488, L13

Schneider, P. C., Günther, H. M., \& Schmitt, J. H. M. M. 2011, A\&A, 530, A123

Schneider, P. C., Günther, H. M., \& Schmitt, J. H. M. M. 2012, A\&A, 542, A123

Shu, F., Najita, J., Ostriker, E., et al. 1994, ApJ, 429, 781

Skinner, S. L., Audard, M., \& Güdel, M. 2011, ApJ, 737, 19

Stelzer, B., Hubrig, S., Orlando, S., et al. 2009, A\&A, 499, 529

Swartz, D. A., Drake, J. J., Elsner, R. F., et al. 2005, ApJ, 628, 811

van Leeuwen, F. 2007, A\&A, 474, 653

Vidal, C. R., Cooper, J., \& Smith, E. W. 1973, ApJS, 25, 37

Vishniac, E. T., \& Brandenburg, A. 1997, ApJ, 475, 263

Wade, G. A., Bagnulo, S., Drouin, D., Landstreet, J. D., \& Monin, D. 2007, MNRAS, 376, 1145

Wassell, E. J., Grady, C. A., Woodgate, B., Kimble, R. A., \& Bruhweiler, F. C. 2006, ApJ, 650, 985

Wise, M. W., Huenemoerder, D. P., \& Davis, J. E. 1997, in Astronomical Data Analysis Software and Systems VI, eds. G. Hunt, \& H. Payne, ASP Conf. Ser., 125, 477

Wisniewski, J. P., Clampin, M., Grady, C. A., et al. 2008, ApJ, 682, 548 